\title{
Educación física en Colombia: formación y tendencias del ejercicio profesional
}

\author{
Physical education in Colombia: formation and trends in professional practice \\ Educação física na Colômbia: formação e tendências na prática profissional \\ MARLUCiO de-SouZa-Martins ${ }^{1}$; SANDRA POSADA-BERNAL ${ }^{2}$ \\ Pontificia Universidad JaVeriana, PUJ, Bogotá, Colombia \\ UNIVERSIDAd SANTO TOMás, USTA, BOGOTÁ, COLOMBIA
}

\begin{abstract}
RESUMEN
Este trabajo tiene como objetivo realizar la revisión de algunos programas curriculares de Educación Física en el contexto europeo y latinoamericano, así como también el panorama colombiano, para la creación de una propuesta de formación profesional en Educación Física en la Pontificia Universidad Javeriana. La formación está centrada en el desarrollo humano y sus dimensiones, por medio de una actitud reflexiva, crítica e investigativa para la construcción del conocimiento y la formación integral. Se observa que en el ámbito europeo y latinoamericano, la tendencia de formación está enmarcada hacia la investigación, el entrenamiento deportivo, la planificación deportiva y la docencia. Por otra parte, el contexto colombiano está orientado hacia la formación de entrenadores deportivos y docentes. Finalmente es necesario diseñar programas de formación que cumplan con las necesidades de país y que permitan dar continuidad a la formación post gradual.
\end{abstract}

Palabras clave: Educación Física. Formación Profesional en Educación Física. Diseño de Programas.

\begin{abstract}
The objective of this work is to review some curricular programs of Physical Education in the European and Latin American context, as well as the Colombian panorama, for the creation of a proposal for a Degree in Physical Education at the Pontificia Universidad Javeriana. The formation of graduates is focused on human development and its dimensions, through a reflective, critical and investigative attitude for the construction of knowledge and comprehensive formation. It is evident that in the European and Latin American context, the formation trend is framed towards research, sports training, sports methodology and teaching. On the other hand, the Colombian context is oriented towards the training of sports coaches and teachers. Finally, it is necessary to design formation programs that meet the needs of the country and allow continuity to post-graduation formation. Keywords: Physical Education. Professional Qualification in Physical Education. Program Design.
\end{abstract}

\section{RESUMO}

Este trabalho tem como objetivo realizar revisão de alguns currículos de Educação Física no contexto europeu e latino-americano, como também no panorama colombiano, para a criação de uma proposta de formação profissional em Educação Física na Pontifícia Universidad Javeriana. A formação está focada no desenvolvimento humano e suas dimensões, através de uma atitude reflexiva, crítica e investigativa para a construção de conhecimento e formação integral. Foi observado que no âmbito europeu e latino-americano, a tendência de formação está centrada em pesquisa, treinamento esportivo, planejamento esportivo e docência. Por outro lado, o contexto colombiano está orientado para a formação de treinadores esportivos e docentes. Finalmente, é necessário projetar programas de formação que atendam às necessidades do país e que permitam a continuidade de formação em pós-graduação.

Palavras-chave: Educação Física. Formação Profissional em Educação Física. Planejamento Curricular.

\footnotetext{
1 Profesor del Departamento de Formación de la Facultad de Educación, PUJ. E-mail: $\frac{\text { mdesouzamartins@javeriana.edu.co }}{2}$ Profesora del Departamento de Humanidades y Formación Integral, USTA. E-mail: sandraposada@usantotomas.edu.co
} 


\section{INTRODUCCIÓN}

Para la Pontificia Universidad Javeriana (PUJ), uno de sus principios fundamentales es la formación integral de las personas, la cual busca el desarrollo armónico de todas las dimensiones del individuo. En este sentido, una de las características principales de la educación jesuita es la Cura Personalis, entendida como el cuidado integral de las personas a través del acompañamiento que se realiza desde el amor y el servicio para que el otro crezca, respetando sus particulares circunstancias y con aprecio a sus capacidades y necesidades (CEPAL, 2017).

De esta manera la formación integral se constituye en una búsqueda constante y sistemática de contribución a la humanización del mundo, a través de la formación de ciudadanos y profesionales educados para propiciar y afrontar los cambios sociales, asumiendo las tensiones desde miradas constructivas y problematizadoras. Lo anterior, a partir de una actitud reflexiva, crítica e investigativa que le permite construir miradas complejas de las realidades y aportar alternativas, propiciando una mayor autonomía moral e intelectual (GÓMEZ et al., 2018).

Este documento presenta la revisión de algunos programas de pregrado en Educación Física a nivel europeo y latinoamericano, así como también a nivel Colombia (nacional y local) donde se plantean aspectos importantes de la formación de docentes como "la implantación y fomento de la educación física, para contribuir a la formación integral de la persona en todas sus edades y facilitarle el cumplimiento eficaz de sus obligaciones como miembro de la sociedad" (MEN, 1995, p. 1).

Por lo anterior, la formación de licenciados materializa la educación física a través de la actividad física y el deporte como objetos de conocimiento para la formación integral de los sujetos. Visto desde la naturaleza social de la educación física y de sus efectos en la vida cotidiana de las personas y de algunas de las problemáticas sociales que tienen que ver con las dificultades de salud, la falta de espacios distintos para el trabajo que se orienten a mejorar las condiciones y la calidad de vida, los problemas a los que se ven expuestos los niños, los jóvenes y adultos por tener pocos espacios para el deporte y la actividad física, son aspectos que se pueden subsanar con la creación de programas enfocados en la necesidades de cada región.

\section{LA EDUCACIÓN FÍSICA EN EUROPA Y AMÉRICA LATINA}

En el contexto mundial la Educación Física se expresa de varias maneras, de acuerdo con el contexto social, cultural y político del país. En este sentido algunos países de oriente, tales como China, Japón y Corea, los Licenciados en Educación Física desarrollan su labor en sociedades marcadas por la corporalidad en las que han diseñado prácticas unidas a su entorno religioso y cultural. De esta manera, el ejercicio de la docencia en Educación Física está fuertemente influenciado por las expresiones artísticas, los juegos populares y comunitarios, así como por las artes marciales milenarias y las corrientes trascendentes como el budismo, el confucionismo y el taoísmo.

A su vez los programas de Licenciatura en Educación Física, en nivel internacional, han tenido otros desarrollos. En el contexto europeo y a partir de la convocatoria del proceso de Convergencia Europea Tuning, el cual examinó la situación actual de formación en Europa en programas de Licenciatura en Educación Física, Actividad Física y Deporte, la Agencia Nacional de Evaluación de la Calidad y Acreditación (ANECA), determinó las competencias y el perfil profesional de los 
egresados en este campo sobre 22 universidades, las cuales otorgan el título de licenciado correspondiente a la denominación del programa (ANECA, 2005). En el siguiente cuadro se presenta la revisión de algunos países en Europa.

Cuadro 1 - Educación Física en Europa, algunos programas seleccionados.

\begin{tabular}{|c|c|c|c|c|c|}
\hline UNIVERSIDAD & PAÍS & CARRERA & CRÉDITOS & AÑNOS & CAMPOS OCUPACIONALES \\
\hline $\begin{array}{c}\text { German Sport } \\
\text { University Cologne } \\
\text { Deutsche } \\
\text { Sporthochschule } \\
\text { Köln } \\
\end{array}$ & Alemania & $\begin{array}{l}\text { Ciencias del } \\
\text { Deporte }\end{array}$ & 240 & 4 & $\begin{array}{l}\text { - Planificación deportiva } \\
\text { - Entrenamiento deportivo } \\
\text { - Investigación en deporte } \\
\text { - Entrenador profesional }\end{array}$ \\
\hline
\end{tabular}

Fuente: Adaptado ANECA (2005).

El cuadro 1, muestra el contexto de la Educación Física en Europa donde se observa que para carreras como Licenciaturas se presentan 180 créditos de formación académica; pero para los programas de Ciencias del Deporte, el número de créditos de formación se encuentra en 240, con una duración de 4 años para ambos programas. En relación con los campos ocupacionales, las características son muy similares en cuanto al campo de actuación; estos programas tienen presente la distinción entre el desarrollo de habilidades deportivas específicas y las metas educativas del entorno escolar, que se concentran desde la perspectiva de la Educación Física en promover que un individuo practique ejercicios a lo largo de su vida.

Por otra parte, en la oferta de programas académicos de Educación Física y carreras afines en América Latina se presenta una situación similar al contexto europeo. Sin embargo, no hay una titulación homogénea y los perfiles y duración de los programas académicos difieren en la mayoría de los países salvo Cuba, Argentina y Brasil.

En Cuba, sólo hay un título académico: Profesional en Cultura Física, Recreación y Deporte, cuyo programa está regulado por el Instituto Nacional de Deportes, Educación Física y Recreación (INDER), órgano superior encargado de planificar, dirigir, racionalizar, divulgar y ejecutar las actividades del deporte, la educación física y la recreación, en todos los niveles y lugares del país. En Argentina, la oferta de programas académicos en el área converge hacia la formación de Licenciados en Educación Física, con dos niveles: a) profesor en educación física, orientado exclusivamente a la docencia en todos los niveles del sistema educativo; b) licenciado, orientado hacia la planeación y organización de servicios relacionados con la actividad física, programación, ejecución y evaluación de procesos de entrenamiento deportivo, investigación, participación en la elaboración de políticas públicas de la actividad física y el deporte.

En Brasil en la oferta de los programas académicos se observan dos titulaciones principales: Graduación en Licenciatura Educación Física y Bachiller (Bacharel) en Educación Física. La primera (Licenciatura) enfatiza la docencia escolar, en cuanto la 
segunda (Bacharel) se encarga de orientar actividades para instituciones públicas o privadas no escolares: clubes, empresas, gimnasios, centros sociales y comunitarios, hoteles, entre otros. En el siguiente cuadro se presenta el contexto en América Latina.

Cuadro 2 - Educación Física en América Latina, algunos programas seleccionados.

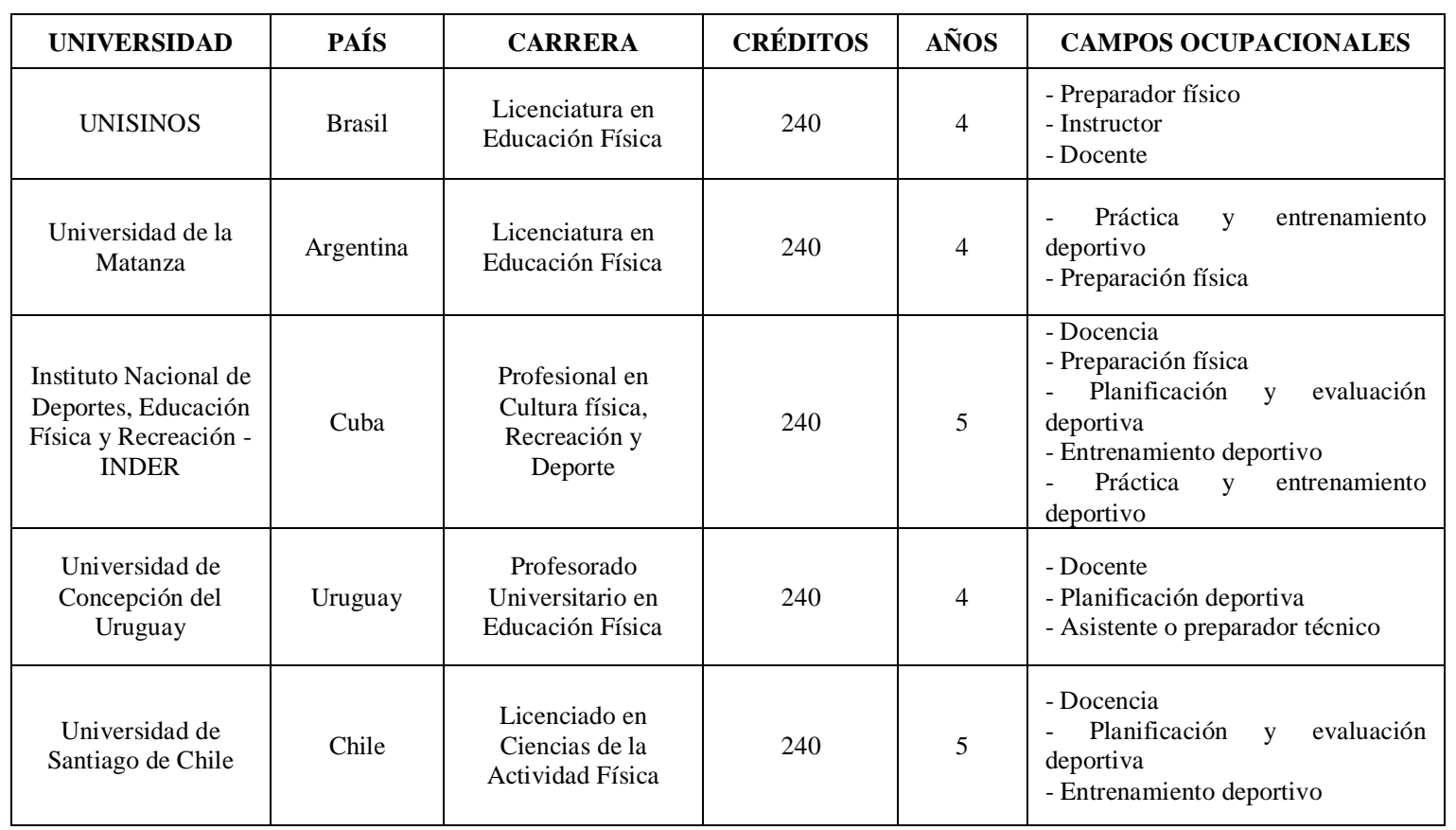

Fuente: Elaboración Propia.

Este panorama latinoamericano, al igual que el europeo, resalta la necesidad de pensar la formación de profesionales en Educación Física con fundamentación en lo disciplinar y en lo pedagógico, y que orienten su labor no sólo a la enseñanza, sino al desarrollo de competencias ocupacionales pertinentes para el contexto actual tales como: emprendimiento, transformación de prácticas sociales y educativas, generación de una conciencia alrededor del cuidado del cuerpo, de sí mismo, y del otro en la perspectiva del desarrollo humano.

\section{LA FORMACIÓN DE EDUCADORES FÍSICOS EN COLOMBIA}

Colombia, ubicada en el extremo noroccidental de América Latina, cuenta con una superficie de $1.141 .748 \mathrm{~km}^{2}$ y una población de 49.032.296 habitantes; está dividido en 6 regiones naturales (Andina, Orinoquía, Caribe, Pacífica, Amazónica e Insular). Su capital Bogotá, se encuentra en la Región Andina y cuenta con 8.080.734 habitantes. Allí es donde más se concentran las instituciones de educación superior, para la formación de profesionales en distintas áreas.

En Colombia el sector de la Educación Física ha tenido un grande crecimiento, lo que se observa en la oferta de los programas de pregrado. Según el estudio Caracterización Ocupacional del Deporte, Recreación y Educación Física, desarrollado por el Servicio Nacional de Aprendizaje (SENA) y el Instituto Colombiano del Deporte (COLDEPORTES), se ofrecían 36 programas de pregrado (SENA; COLDEPORTES, 2016), y según la Asociación Red Colombiana de Facultades de Deporte, Educación Física y Recreación (ARCOFADER), en 2007 había 55 programas nacionales de pregrado (ARCOFADER, 2017). Esto ha creado la necesidad de desarrollar 
estructuradamente el área de conocimiento y de fortalecer la formación de licenciados, que desde una mirada educativa, pedagógica y didáctica aporten propuestas orientadas al desarrollo de capacidades y la construcción de conocimientos dentro de campo de la Educación Física, la Actividad Física y el Deporte.

Con base en la revisión general realizada en el Sistema Nacional de Información de la Educación Superior (SNIES, 2018), se encontraron los siguientes hallazgos transversales que brindan una mirada panorámica de la formación profesional en el sector de la Educación Física en Colombia:

- Actualmente, según registro de SNIES (2018), existen 44 Instituciones de Educación Superior (IES) que brindan programas relacionados con la denominación Licenciatura en Educación Física y, en Bogotá, se encuentran 12 programas de los cuales 6 tienen acreditación de alta calidad.

- La denominación de los Programas muestra una gran variedad, es así como se cuenta con 21 nombres oficiales relacionados con la denominación: educación física, actividad física, deporte, y recreación, entre los principales.

- En relación con el número de créditos ofrecidos, se tiene actualmente un rango entre 38 y 181, con un promedio de 140 créditos para el país. Estos créditos están distribuidos en un mínimo de 1 año y máximo de 5.5 años, teniendo en cuenta que estos programas ofrecen titulación como Tecnólogos, Licenciados y Profesionales.

- La denominación al otorgar el título profesional en educación física, actividad física, deporte, y recreación, se encuentran discriminadas así: Tecnólogo (11\%), Licenciado (62\%) y "Profesional en..." (27\%).

- A pesar del relativo alto número de programas e IES, que imparten los estudios del sector en Colombia, existen a nivel nacional 26 Programas con Acreditación de Alta Calidad, lo cual representa tan solo un $41 \%$ del total del país.

De otra parte en el SNIES (2018), se encuentran registrados en la oferta nacional un total de 64 programas en Educación Física y afines, de los cuales 7 corresponden a formación Tecnológica, 44 a Licenciaturas y 17 a títulos Profesionales. El 56\% de los programas se ubican en la Región Andina en donde el 19\% de los programas corresponde a capital del País (Bogotá). El 44\% restante de los programas, están distribuidos entre la Región Pacífica 25\%, Caribe 15\%, y $4 \%$ en otras regiones. Por otra parte, se observa que la duración más común es de 5 años para un promedio de 171 créditos.

En relación con la organización de los programas, de acuerdo con su titulación y orientación, en el SNIES (2018) se encuentra que la formación en el campo de la Educación Física, Recreación y Deporte ocupa el primer lugar con un $44.7 \%$ de los programas orientados a estas temáticas; seguido se encuentra el Deporte $17 \%$ y la Educación Física y Deporte $14.9 \%$.

A continuación, se presenta un cuadro comparativo general de programas de Educación Física y campos afines en ámbito nacional (Cuadro 3) y en la ciudad de Bogotá (Cuadro 4), con base en los datos registrados en el SNIES en mayo de 2018. Se relaciona información con base en la denominación del programa, el número de créditos, los años de duración, y los campos ocupacionales.

Cuadro 3 - Educación Física en Colombia (Nacional), algunos programas seleccionados.

\begin{tabular}{|c|c|c|c|c|}
\hline UNIVERSIDAD & CARRERA & CRÉDITOS & AÑOS & CAMPOS OCUPACIONALES \\
\hline $\begin{array}{c}\text { Universidad de San } \\
\text { Buenaventura }\end{array}$ & $\begin{array}{c}\text { Licenciatura en Educación Física } \\
\text { y Deporte }\end{array}$ & 160 & 5 & $\begin{array}{l}\text { - Sistema educativo } \\
\text { - Entrenador deportivo }\end{array}$ \\
\hline
\end{tabular}




\begin{tabular}{|c|c|c|c|c|}
\hline $\begin{array}{c}\text { Fundación Universitaria } \\
\text { Juan de Castellanos }\end{array}$ & $\begin{array}{c}\text { Licenciatura en Educación } \\
\text { Física, Recreación y Deporte }\end{array}$ & 175 & 5 & $\begin{array}{l}\text { - Sistema educativo } \\
\text { - Entrenamiento deportivo }\end{array}$ \\
\hline $\begin{array}{c}\text { Universidad Católica de } \\
\text { Oriente }\end{array}$ & $\begin{array}{c}\text { Licenciatura en Educación } \\
\text { Física, Recreación y Deporte }\end{array}$ & 164 & 5 & - Sistema educativo \\
\hline $\begin{array}{c}\text { Universidad Autónoma } \\
\text { del Caribe- } \\
\text { Uniautonoma }\end{array}$ & Deporte y Cultura Física & 150 & 5,5 & $\begin{array}{l}\text { - Entrenamiento deportivo } \\
\text { - Salud }\end{array}$ \\
\hline $\begin{array}{c}\text { Corporación } \\
\text { Universitaria } \\
\text { Latinoamericana - CUL }\end{array}$ & $\begin{array}{c}\text { Licenciatura en Educación } \\
\text { Básica con énfasis en Educación } \\
\text { Física, Recreación y Deporte }\end{array}$ & 160 & 5 & Sistema educativo \\
\hline
\end{tabular}

Fuente: Elaboración propia.

En la oferta académica nacional se evidencia una diferencia en la denominación de los programas, con una predominancia hacia la Recreación y el Deporte, manteniendo el enfoque pedagógico de las licenciaturas. Esto implica que el campo ocupacional de estos programas, se orienta al entrenamiento deportivo, como también a la labor docente en el contexto educativo. Por otra parte, en términos del número de créditos académicos y duración de los programas es similar con un promedio de 4,9 años y 161,8 créditos.

Cuadro 4 - Educación Física en Colombia (Bogotá), algunos programas seleccionados.

\begin{tabular}{|c|c|c|c|l|}
\hline UNIVERSIDAD & CARRERA & CRÉDITOS & AÑOS & CAMPOS OCUPACIONALES \\
\hline $\begin{array}{c}\text { Universidad de Santo Tomás } \\
\text { (USTA) }\end{array}$ & $\begin{array}{c}\text { Cultura Física Recreación y } \\
\text { Deporte }\end{array}$ & 146 & 5 & $\begin{array}{l}\text { - Administración deportiva } \\
\text { - Salud }\end{array}$ \\
\hline $\begin{array}{c}\text { Universidad Manuela Beltrán } \\
\text { UMB }\end{array}$ & Ciencias del Deporte & 167 & 5 & - Entrenamiento deportivo \\
\hline Universidad Libre & $\begin{array}{c}\text { Licenciatura en Educación } \\
\text { Básica con énfasis en Educación } \\
\text { Física, Recreación y Deportes }\end{array}$ & 170 & 5 & - Sistema educativo \\
\hline Universidad UDCA & Ciencias del Deporte & 180 & 5 & - Entrenamiento deportivo \\
\hline Universidad INCCA & Ciencias del Deporte & 161 & 5 & - Entrenamiento deportivo \\
\hline
\end{tabular}

Fuente: Elaboración propia.

La información anterior muestra que existen diferencias marcadas entre los distintos programas a nivel de los campos ocupacionales, pues la oferta varía entre aquellos que orientan sus currículos a la salud y la administración deportiva (USTA), la educación básica en educación física (Libre), las ciencias aplicadas al deporte (UDCA y UMB). Las denominaciones tienden a dos grupos: Licenciados en Educación Física y Profesionales en Ciencias del Deporte y la Cultura Física. En cuanto al número de créditos, existe un margen de diferencia muy pequeña entre 146 y 180 créditos en todos los programas, manteniéndose los 5 años de formación profesional.

Con base en los datos del Observatorio Laboral para la Educación (OLE, 2018), para el año 2017 se reporta un total de 1267 graduados correspondientes a los 12 programas ofertados en la ciudad de Bogotá, siendo el 30\% (378) representan las instituciones oficiales y el $70 \%$ (889) pertenecen al sector privado. Al realizar un análisis sobre el ingreso que obtienen los graduados de estos programas se encuentra que reciben en el promedio un salario mensual de USD 500 para egresados del sector oficial; para el caso del sector privado el salario promedio mensual es de USD 550, para empleos correspondientes al área educativa.

Por otro lado, al revisar la información de otras áreas relacionadas con la empleabilidad se encuentra que el egresado puede también realizar labores en espacios distintos al educativo como los servicios sociales y de salud y actividades de servicios comunitarios, sociales y personales. De acuerdo con la información anterior, los egresados pueden trabajar además del sector educativo, en clubes, ligas, gimnasios, 
cajas de compensación familiar y demás entidades privadas o estatales interesadas en contratar personal formado en el área (GÓMEZ et al., 2018). En este sentido, el promedio de ingreso se incrementa al combinar la actividad docente en distintos escenarios del contexto escolar.

Esta perspectiva de formación del licenciado, de acuerdo con las necesidades de la sociedad, invita a las universidades a formar sujetos críticos, con una amplia base disciplinar y socio humanística que puedan tener un amplio impacto en sus entornos particulares, sin limitar su labor a un saber instrumental. Es desde este contexto donde el Programa de Licenciatura en Educación Física que presenta la Pontificia Universidad Javeriana tiene un alto nivel de pertinencia y relevancia para la formación de profesionales en este campo, que aporten al desarrollo del campo y a la educación de manera específica (GÓMEZ et al., 2018).

\section{LA PROPUESTA DE FORMACIÓN DE LICENCIADOS EN EDUCACIÓN FÍSICA PARA LA PONTIFICIA UNIVERSIDAD JAVERIANA}

Como parte del proceso de planeación estratégica de la Facultad de Educación y en el marco de la reforma normativa con respecto a la oferta de programas de formación docente, en especial la Resolución 18583 del Ministerio de la Educación (MEN, 2017), se retomaron iniciativas previas para el diseño de un programa de Licenciatura en Educación Física con el propósito de formar licenciados en el campo de la Educación Física con sólidas bases disciplinares y altas capacidades pedagógicas y didácticas, desde una perspectiva investigativa e innovadora.

En este sentido, la denominación de esta propuesta se hace con base en la Resolución 18583 de septiembre 15 de 2017 del Ministerio de Educación Nacional (MEN, 2017), que en su Artículo 2 - Numeral 1, hace referencia a la denominación de las licenciaturas, para este caso, "Licenciatura en Educación Física", correspondiente al área obligatoria y fundamental Educación Física, Recreación y Deportes. De esta forma, el objeto de estudio del programa se marca en la Educación Física, en relación con la Actividad Física y el Deporte, integrando aportes provenientes de las Ciencias Biológicas, Sociales y Humanas, los cuales se resignifican desde la reflexión en la práctica educativa.

Con este contexto y con base en la naturaleza y ser de la Pontificia Universidad Javeriana, se diseñó una propuesta de formación de licenciados cuyos contenidos curriculares están organizados desde una perspectiva de problematización, en cuatro grandes componentes para ser ofertados a lo largo de 4 años y 160 créditos (GÓMEZ et al., 2018):

- Fundamentos generales, pedagógicos y misionales (56 créditos). Este componente aborda los espacios académicos para el desarrollo del pensamiento educativo y pedagógico desde una perspectiva integradora, así como aquellos orientados al conocimiento y la reflexión de los elementos identitários y misionales javerianos. Estos espacios contribuyen a la formación de licenciados desde una perspectiva integral, en clave interdisciplinar.

- Fundamentos de saberes disciplinares y didácticas de la disciplina (48 créditos). Se abordan los fundamentos conceptuales de la disciplina y las perspectivas didácticas para la enseñanza y aprendizaje de la misma. Ubican la Educación Física como objeto de conocimiento especializado del programa, en relación con dos objetos de conocimiento asociados: la actividad física y el deporte, los cuales se conciben de manera complementaria. Lo anterior, en una 
relación dialogante con la pregunta por los procesos formativos, de enseñanza y de aprendizaje que conllevan tales objetos de estudio.

- Práctica Pedagógica (40 créditos). Este componente se constituye en el centro de la formación de los licenciados, pues en ella convergen y se resignifican los fundamentos educativos, pedagógicos, didácticos y disciplinares, en diálogo constante con las experiencias y saberes propios de los diversos contextos formativos.

- Electivo (16 créditos). Este componente aporta a la formación integral del estudiante, tanto en su ser como licenciado, como en su ser humano.

En el contexto nacional, éste programa busca aportar al desarrollo prospectivo de la Educación en el país y sus principales metas. Con base en lo establecido en el Plan Decenal de Educación 2016 - 2026 (MEN, 2016) y sus 10 desafíos estratégicos constitutivos, para contribuir con el desarrollo de País, los egresados de este programa serán profesionales reflexivos, críticos y comprometidos socialmente, capaces de liderar proyectos educativos, escolares y no escolares, con énfasis en la dimensión corporal, con base en su sólida formación pedagógica y disciplinar en el campo de la educación física, la actividad física y el deporte.

\section{CONSIDERACIONES FINALES}

Teniendo en cuenta la importancia de la formación de docentes y en particular en Educación Física, Actividad Física y Deportes, cobra sentido la revisión de distintas apuestas de formación tanto a nivel internacional como nacional. En este sentido, considerar las distintas propuestas curriculares y objetivos de formación en el ámbito europeo y latinoamericano en donde la tendencia está enmarcada en una formación hacia la investigación, el entrenamiento deportivo, la planificación deportiva y la docencia. Por otra parte, el contexto colombiano está orientado hacia la formación de entrenadores deportivos y docentes.

De esta forma, la propuesta del programa de Licenciatura en Educación Física de la PUJ es innovadora, pues asume una perspectiva interdisciplinar e investigativa, que contempla prácticas pedagógicas escolares y no escolares en clave inclusiva $y$ emprendedora; así mismo, articula la participación de otros Departamentos de la Universidad; también, asume la formación en inglés tanto en espacios específicos para el aprendizaje del idioma como en el marco de las asignaturas de los componentes pedagógicos y disciplinares y cuenta con condiciones de infraestructura excepcionales. Adicionalmente, contempla y refleja los valores y razón de ser de los egresados Javerianos, hacia la formación el cuidado integral de las personas en distintos ámbitos.

Dado que en el contexto académico la formación post gradual es fundamental, los egresados de este programa contarán con la posibilidad de hacer una Maestría en Educación, ofertada por la Facultad de Educación de la PUJ, en la línea de Actividad Física, Salud y Deporte. De igual manera, pueden optar por las diversas ofertas de Maestrías a nivel local y nacional en las áreas de Educación Física, Actividad Física, Deporte y Recreación. Sin embargo, los egresados que quisieran iniciar su proceso de formación doctoral, deben realizarlo fuera del país ya que en Colombia hasta el momento no existe un Programa de Doctorado en estas áreas. Este panorama nos lleva a reflexionar sobre la importancia de diseñar programas que continúen la línea de formación: pregrado, maestría y doctorado, que estén orientados a solucionar las problemáticas de país y con una visión holística e interdisciplinar hacia la internacionalización. 


\section{REFERENCIAS}

ANECA. Agencia Nacional de Evaluación de la Calidad y Acreditación. Libro blanco: título de grado en ciencias de la actividad física $y$ el deporte. 2005. Disponible en: < http://www.aneca.es/var/media/150280/libroblanco_humanidades_def.pdf>. Recuperado el: 10 ago. 2017.

ARCOFADER. Asociación Red Colombiana de Facultades de Deporte, Educación Física y Recreación. Exámenes de calidad de la educación superior en educación física, deporte, recreación y afines. Disponible en:

http://www.renovacionmagisterial.org/inicio/docs/200906/docenteseducacionfisica.pdf>. Recuperado el: 10 ago. 2017.

CEPAL. Conferencia de Provinciales Jesuitas de América Latina. La cura personalis en la educación jesuita. Disponible en: < https://cpalsocial.org/2189.html>. Recuperado el: 22 ago. 2017.

GÓMEZ, F. A.; ZULUAGA, Z. P.; SARMIENTO, J. H.; SANDOVAL, F. J.; DE-SOUZA-MARTINS, M; FlÓREZ, A. A.; POSADA-BERNAL, S.; TARAZONA, A. E. Programa de licenciatura en educación física: documento maestro para solicitar el registro calificado. Bogotá: Facultad de Educación, Pontificia Universidad Javeriana, 2018.

MEN. Ministerio de Educación Nacional. Ley 181 del 18 de Enero de 1995. 1995. Disponible en: < https://www.mineducacion.gov.co/1621/articles-85919_archivo_pdf.pdf>. Recuperado el: 20 abr. 2017.

MEN. Ministerio de Educación Nacional. Plan Decenal de Educación 2016 - 2026. 2016. Disponible en:

http://www.plandecenal.edu.co/cms/images/PLAN\%20NACIONAL\%20DECENAL\%20DE\%20EDUCA CION\%202DA\%20EDICION_271117.pdf>. Recuperado el: 20 abr. 2017.

MEN. Ministerio de Educación Nacional. Resolución 18583 del 15 de Septiembre de 2017. 2017. Disponible en:

https://www.usbcali.edu.co/sites/default/files/resolucion_final_18583_de_2017deroga_2041.pdf $>$. Recuperado el: 1 de feb. 2018.

OLE. Observatorio Laboral para la Educación. Cifras del observatorio 2017. Disponible en: < http://www.graduadoscolombia.edu.co/html/1732/w3-article-344798.html>. Recuperado el: 1 sept. 2018.

SENA; COLDEPORTES. Servicio Nacional de Aprendizaje e Instituto Colombiano del Deporte. Caracterización ocupacional del deporte, recreación y educación física. 2016. Disponible en: < https://repositorio.sena.edu.co/bitstream/11404/2105/1/3019.pdf>. Recuperado el: 1 sept. 2017.

SNIES. Sistema Nacional de Información de la Educación Superior. Consulta instituciones de educación superior. Disponible en: < https://www.mineducacion.gov.co/sistemasinfo/Sistemas-deInformacion-en-Ed-Superior-especializados/214140:SACES-Sistema-de-Aseguramiento-de-la-Calidadde-Educacion-Superior>. Recuperado el: 20 mayo 2018.

Recibido en: 30 oct. 2018.

Aprobado en: 23 dic. 2018. 